\title{
Preliminary Report on Empirical Study of Repeated Fragments in Internal Documentation
}

\author{
Milan Nosál', Jaroslav Porubän \\ Department of Computers and Informatics, \\ Faculty of Electrical Engineering and Informatics, \\ Technical University of Košice \\ Letná 9, 042 00, Košice, Slovakia \\ Email:milan.nosal@gmail.com, jaroslav.poruban@tuke.sk
}

\begin{abstract}
In this paper we present preliminary results of an empirical study, in which we used copy/paste detection (PMD CPD implementation) to search for repeating documentation fragments. The study was performed on 5 open source projects, including Java 8 SDK sources. The study shows that there are many occurrences of copy-pasting documentation fragments in the internal documentation, e.g., copy-pasted method parameter description. Besides these, many of the copy-pasted fragments express some domain or design concern, e.g., that the method is obsolete and deprecated. Therefore the study indicates that the cross-cutting concerns are present in the internal documentation in form of documentation phrases.
\end{abstract}

\section{INTRODUCTION}

$\mathbf{P}$ RESERVING and comprehending developer's concerns (intents) in the source code is still a current challenge in software development [1], [2], [3], [4]. In this paper we analyze the internal documentation (source code comments, JavaDoc, etc.) to recognize repeating documentation fragments that document those concerns (or features [5]). Our research question for this work is: Does internal documentation contain significant duplication? To answer this question we performed a copy/paste detection study, in which we analyzed JavaDoc comments in 5 open source projects. In this report we present preliminary results that indicate that there is a significant duplication of text in internal documentation. These repeating documentation fragments constitute documentation phrases discussed in several works - e.g., our previous work [6], or the one by Horie et al. [7]. This way the study has a potential to highlight the importance of those works and it may stimulate further attention to this topic.

\section{Documentation Phrases}

A documentation phrase is a set of documentation fragments with the same or similar formulation (part of sentence, sentence, a set of sentences) that can be found across the software system or even across multiple systems. Documentation fragments that represent the same documentation phrase usually document the same domain or design property that is shared by the documented program elements [6]. Horie et al. [7] likened the documentation phrases to crosscutting concerns from aspect oriented programming (AOP [8]).

As an example we can use the Swing library for component graphical user interfaces in Java. The library is not thread safe and therefore the programmer has to pay extra caution when using it in multithreaded systems (she has to use Event Dispatch Thread to safely work with the Swing components). Swing JavaDoc documents it and for each affected class includes a warning (see JPanel documentation in Figure 1).

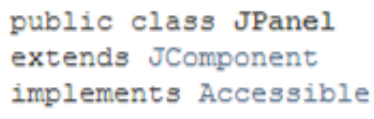

JPanel is a generic lightweight container. For examples and task-orier documentation for JPanel, see How to Use Panels, a section in The Ja Tutorial.

Warning: Swing is not thread safe. For more information see Swing's Threading Policy.

Warning: Serialized objects of this class will not be compatible with fut Swing releases. The current serialization support is appropriate for she term storage or RMI between applications running the same version of Swing. As of 1.4, support for long term storage of all JavaBeans ${ }^{\mathrm{TM}}$ has added to the java.beans package. Please see XMLEncoder.

Fig. 1. A NotThreadSafe documentation phrase instance in the Swing JPanel documentation

For this warning to be included in the JavaDoc, its HTML snippet has to be copy-pasted in the JavaDoc comment of each affected class.

\section{Documentation Copy/PAste Study}

In this study we analyzed the internal documentation of several Java frameworks and libraries to detect currently existing documentation phrases. In order to find documentation phrases, we performed a copy/paste detection ${ }^{1}$ on the documentation.

We have modified the PMD Copy/Paste Detection (CPD) tool $^{2}$ to support copy/paste detection on JavaDoc documentation. The tool was fed preprocessed sources of several libraries and open source projects in Java and it analysed them to detect duplications in documentation that would indicate the potential

\footnotetext{
${ }^{1}$ Copy/paste detection is usually used to search for code that needs to be refactored, or to detect plagiarism [9].

${ }^{2} \mathrm{http}: / /$ pmd.sourceforge.net/
} 

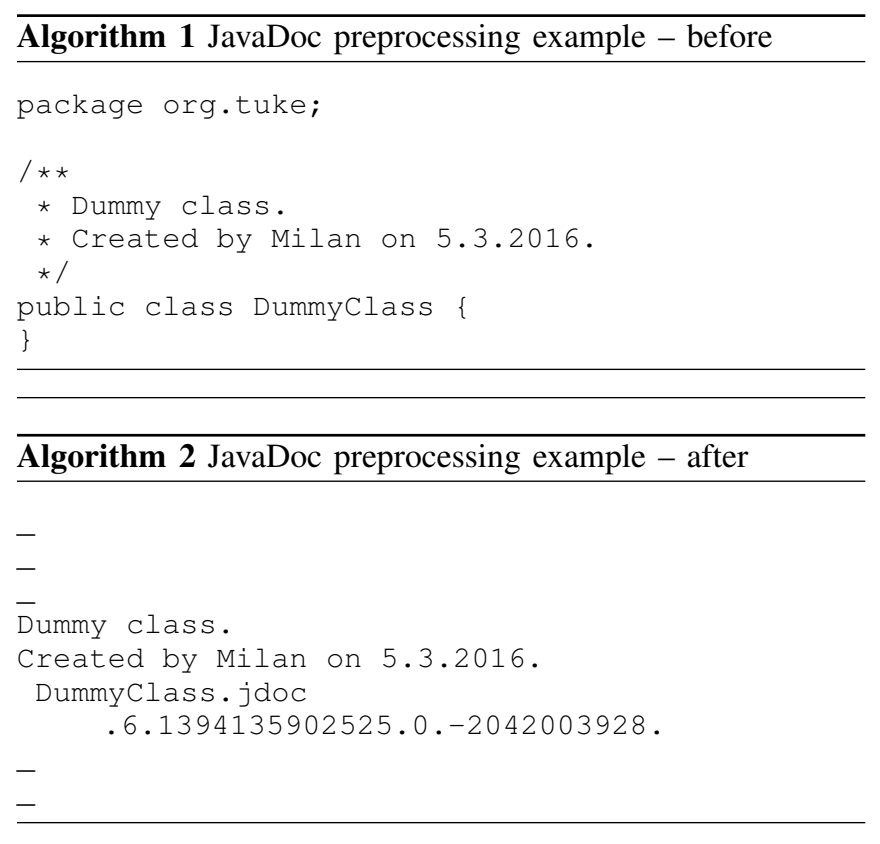

documentation phrases. We will discuss the process phases in more details in following sections.

\section{A. Java Sources Preprocessing}

In our experiment we used the tool to detect simple nonparametrized documentation phrases in the JavaDoc documentation. However, the PMD CPD was designed to be a code analysis tool and as such its purpose was to detect duplication in programming languages. Documentation phrases are fragments of documentation in a natural language.

PMD CPD tool works with language lexical tokens that it compares to detect duplications in their usage. To reduce tokenization complexity we pre-processed the sources to remove all the characters and tokens that are not the documentation. In other words the preprocessed sources are source files with only JavaDoc comments in their comments. Let us consider a simple class with JavaDoc from Listing 1.

This source file would be transformed to the content presented in Listing $2^{3}$

Java lexical tokens were discarded along with asterisks indicating that following lines are part of JavaDoc (lines are preserved for backtracking to original sources). At the end of each JavaDoc comment we added a randomly generated unique "anchor" (DummyClass.jdoc.6.1394135902525

.0.-2042003928. in the example) that prevented detection of duplicates spanning multiple comments.

\section{B. PMD CPD Modification}

PMD CPD tool uses a tokenizer to read files and obtain lexical tokens of the language. In our experiment we used our custom tokenizer that divided the preprocessed files into sentences. Each sentence in the file was a single token. If we

\footnotetext{
${ }^{3}$ We used underscores here to highlight empty lines.
}

consider the DummyClass example from section III-A, the tokenizer would return following three tokens:

- "Dummy class."

- "Created by Milan on 5.3.2016."

• "DummyClass.jdoc.6.1394135902525.0.-2042003928."

Separators for the tokenization were characters '., '?', and '!' followed by a whitespace character (therefore the date in the second token from the example was not divided in multiple tokens), or a new line character followed by an empty line.

\section{Document Phrases Detection}

For the duplication detection process we used the standard PMD CPD implementation (according to http:// pmd.sourceforge.net/pmd-4.3.0/cpd.html they use Karp-Rabin string matching algorithm). We registered our modification in LanguageFactory and GUI classes and used the graphical user interface provided by the GUI class to run the tool.

In the setup of the copy/paste detection we set the 'Report duplicate chunks larger than:' option to a single token. This way PMD CPD reported even duplication of a single token a single line in the documentation. The results were serialized as XML so that we could use XPath with XSLT to process them. First post-processing removed all the results that did not have at least 4 duplications - we considered 4 instances of a documentation phrase a reasonable threshold for considering it a significant documentation phrase.

\section{RESULTS}

We performed the experiment on the following open source Java projects:

- sources of Java 8 standard edition ${ }^{4}$ with 7703 source files,

- PicoContainer ${ }^{5}$ with 1067 source files,

- JasperReports library ${ }^{6}$ with 2834 source files,

- JoSQL ${ }^{7}$ (SQL for Java Objects) with 85 source files, and

- $\mathrm{jEdit}^{8}$ with 573 source files.

\section{A. Copy/paste Detection Results}

The PMD CPD tool discovered 6102 duplicated fragments of various lengths in Java 8 source code. 2221 of them were duplicated fragments that had 4 instances. The highest number of instances of a single duplicated fragment was 344. Second highest were two duplicated fragments both with 299 instances. Figure 2 shows an overview of obtained results. We will provide a more detailed analysis of these data below.

PicoContainer contained 70 duplicated fragments ranging from 36 duplicated fragments with 4 instances to a single fragment duplicated 634 times. Figure 2 presents results for PicoContainer in a simple chart. Closer inspection of the results showed that duplicated fragments with the highest

\footnotetext{
${ }^{4}$ http://www.oracle.com/technetwork/java/javase/downloads/ java-archive-javase8-2177648.html, JDK version $8 \mathrm{u} 20$

${ }^{5} \mathrm{https} / / /$ github.com/picocontainer/picocontainer, commit $0 \mathrm{f} 8172 \mathrm{~b}$

${ }^{6} \mathrm{http} / / /$ sourceforge.net/projects/jasperreports/files/jasperreports/, version 6.0 .0

${ }^{7}$ http://sourceforge.net/projects/josql/files/josql/, version 2.2

${ }^{8} \mathrm{http}: / /$ sourceforge.net/projects/jedit/files/jedit/, version 5.2.0
} 


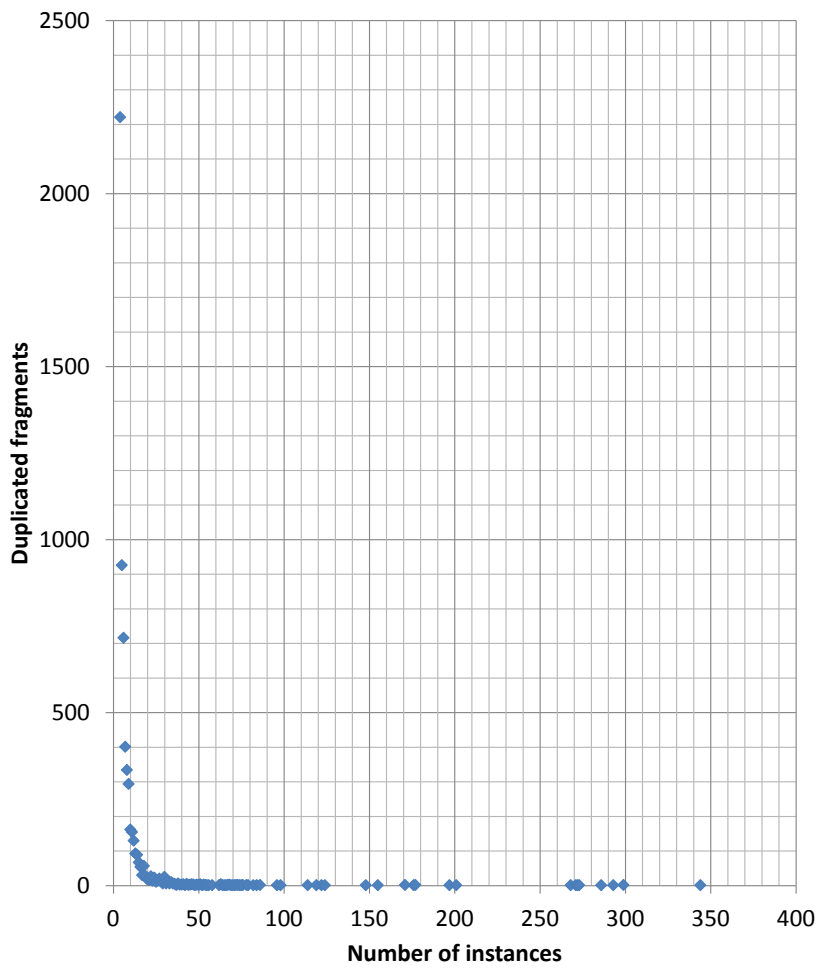

Fig. 2. Duplicated fragments detected by PMD CPD in standard Java

numbers of instances were just lines consisting of asterisks (*) probably used as a visual separator in the documentation.

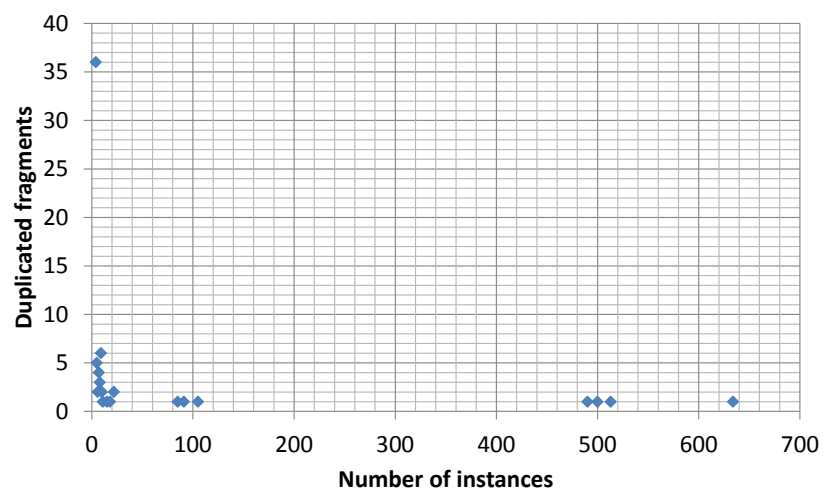

Fig. 3. Duplicated fragments detected by PMD CPD in PicoContainer sources

JasperReports contained 131 duplicated fragments ranging from 44 duplications with 4 instances to a single duplicated fragment with 106 instances. Figure 4 presents results for JasperReports in a simple chart. In this case the duplicated fragment with 106 instances was a documentation phrase reporting that the documented program elements were deprecated and to be removed: '@deprecated To be removed.'. Deprecation naturally expresses design concern - the given program element became obsolete and should not be used anymore.

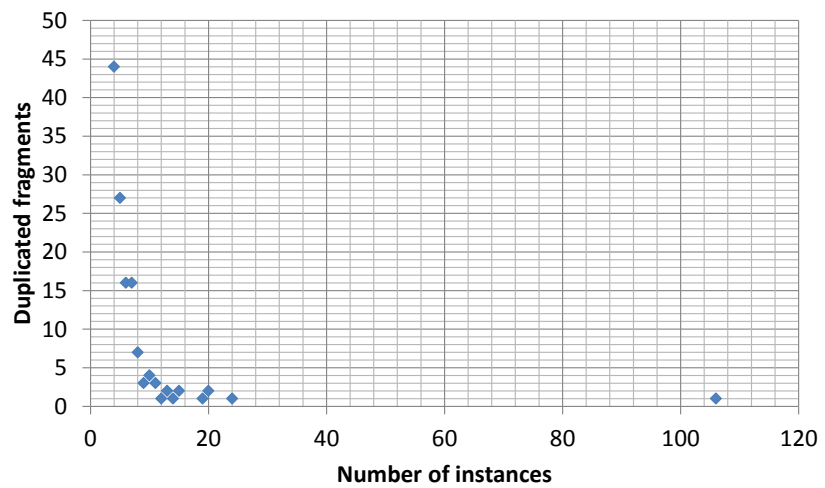

Fig. 4. Duplicated fragments detected by PMD CPD in JasperReports sources

In JoSQL the PMD CPD discovered 31 duplicated fragments. The most 'potent' duplicated fragment with 54 instances was a paramater description: '@param q The Query object.'. Parameter descriptions, especially this simple, could hardly be considered reasonable documentation phrases. The rest of results can be seen in Figure 5.

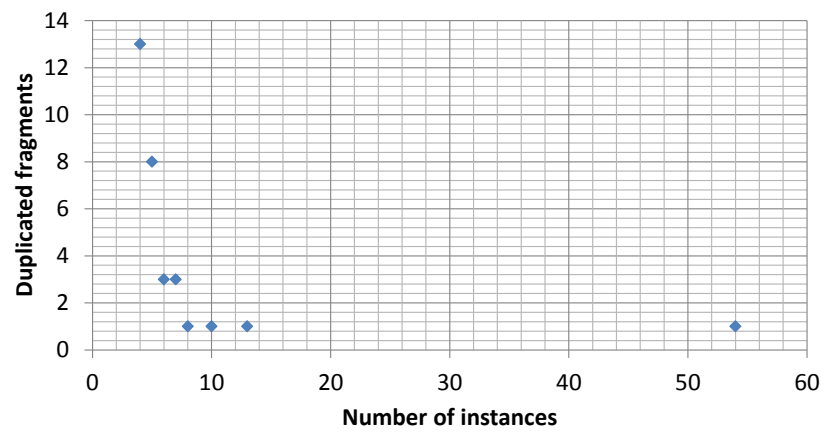

Fig. 5. Duplicated fragments detected by PMD CPD in JoSQL sources

jEdit project sources manifested 76 duplicated fragments with 4 or more instances. Again, the most 'potent' duplicated fragment (with 78 instances) was a line of asterisks. However, the second most 'potent' duplicated fragment was a sentence reporting that the documented method is thread-safe: 'This method is thread-safe.', thus showing that the thread-safe documentation phrase would be useful even beyond the scope of standard Java sources. The results overview can be seen in Figure 6.

We can conclude that duplicated fragments (documentation phrases) are common in practice.

\section{Threats to VALIDITY}

We should mention several threats to validity that should to be considered for this study. First, the copy/paste detection found all the duplicated sentences in the documentation, even those that could hardly be assigned a concern. In those 


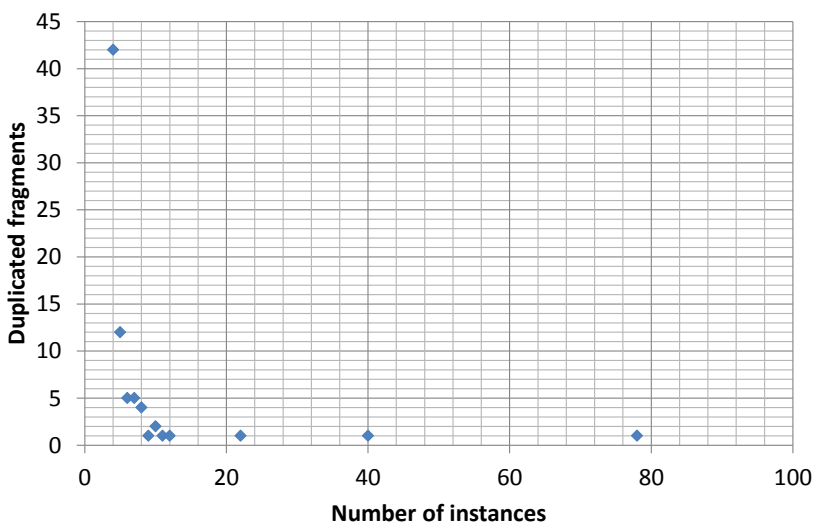

Fig. 6. Duplicated fragments detected by PMD CPD in jEdit sources

cases using documentation weaving [7] would be impractical. Further examination would be useful.

Second, the modified PMD CPD detected solely static phrases - fragments of the documentation that were copy/pasted in documentation. Inclusion of parametrized documentation phrases [6] would be welcome.

\section{RELATED WORK}

Maalej et al. in [10] present a study of knowledge patterns in API reference documentation. They define patterns as knowledge types that categorize types of information expressed by a particular documentation unit (a fragment of API documentation documenting one API element). As example we can mention types like the Functionality and Behavior knowledge type that describes what the API does or the Code Examples that provides a code sample showing how to use the API.

The work of Horie et al. [7] discusses documentation phrases from the aspect-oriented viewpoint. They view documentation phrases as cross-cutting concerns. Their tool CommentWeaver is able to weave documentation phrases the same way as advices are woven in aspect-oriented programming. Our work presented in [6] continues in their work. There we propose using source code annotations to indicate program elements that should be documented by a given documentation phrase.

Shi et al. in [11] present an empirical quantitative study of API documentation evolution. They analyze the documentation to detect which parts of documentation are frequently revised, how often these revisions indicate behavioral changes in API and how often do these revisions occur. The contribution of their work is in emphasizing the importance of API documentation evolution in order to prevent defects in software using the given API.

\section{CONCLUSION}

In conclusion, the presented results underline the significance of approaches like the one we presented in [6], or the one by Horie et al. [7], which centralize the management of such a documentation phrase into one place and thus ease their maintenance and evolution.
In the future work we need to further examine the results and confirm the significance of fragments that can be considered a concern (intent). An interesting modification of the experiment would be inclusion of the parametrized fragments as well.

\section{ACKNOWLEDGMENT}

This work was supported by project KEGA No. 019TUKE4/2014 Integration of the Basic Theories of Software Engineering into Courses for Informatics Master Study Programmes at Technical Universities - Proposal and Implementation.

\section{REFERENCES}

[1] V. Vranić, J. Porubän, M. Bystrický, T. Frt'ala, I. Polášek, M. Nosál', and J. Lang, "Challenges in preserving intent comprehensibility in software," Acta Polytechnica Hungarica, vol. 12, no. 7, pp. 57-75, 2015. doi: 10.12700/aph.12.7.2015.7.4. [Online]. Available: http://dx.doi.org/10.12700/aph.12.7.2015.7.4

[2] J. Kollár, M. Sičák, and M. Spišiak, "Towards Machine Mind Evolution," in 2015 Federated Conference on Computer Science and Information Systems, ser. FedCSIS 2015, Sept 2015. doi: 10.15439/2015F210 pp. 985-990. [Online]. Available: http://dx.doi.org/10.15439/2015F210

[3] J. Juhár and L. Vokorokos, "A review of source code projections in integrated development environments," in 2015 Federated Conference on Computer Science and Information Systems, ser. FedCSIS 2015, Sept 2015. doi: 10.15439/2015F289 pp. 923-927. [Online]. Available: http://dx.doi.org/10.15439/2015F289

[4] E. Pietriková and S. Chodarev, "Profile-driven source code exploration," in 2015 Federated Conference on Computer Science and Information Systems, ser. FedCSIS 2015, Sept 2015. doi: 10.15439/2015F238 pp. 929-934. [Online]. Available: http://dx.doi.org/10.15439/2015F238

[5] R. Táborský and V. Vranić, "Feature Model Driven Generation of Software Artifacts," in 2015 Federated Conference on Computer Science and Information Systems, ser. FedCSIS 2015, Sept 2015. doi: 10.15439/2015F364 pp. 1007-1018. [Online]. Available: http: //dx.doi.org/10.15439/2015F364

[6] M. Nosál' and J. Porubän, "Reusable software documentation with phrase annotations," Central European Journal of Computer Science, vol. 4, no. 4, pp. 242-258, 2014. doi: 10.2478/s13537-014-0208-3 [Online]. Available: http://dx doi.org/10.2478/s13537-014-0208-3

[7] M. Horie and S. Chiba, "Tool Support for Crosscutting Concerns of API Documentation," in Proceedings of the 9th International Conference on Aspect-Oriented Software Development, ser. AOSD '10. New York, NY, USA: ACM, 2010. doi: 10.1145/1739230.1739242. ISBN 978-1-60558-958-9 pp. 97-108. [Online]. Available: http: //dx.doi.org/10.1145/1739230.1739242

[8] V. Vranić and B. Kuliha, "Realizing changes by aspects at the design level," in Proceedings of the 2015 IEEE 19th International Conference on Intelligent Engineering Systems, ser. INES 2015, Sept 2015. doi: 10.1109/INES.2015.7329736 pp. 369-374. [Online]. Available: http://dx.doi.org/10.1109/INES.2015.7329736

9] J. Genči, About One Way to Discover Formative Assessment Cheating. Cham: Springer International Publishing, 2015, pp. 83-90. ISBN 978-3-319-06764-3. [Online]. Available: http://dx.doi.org/10. 1007/978-3-319-06764-3 11

[10] W. Maalej and M. P. Robillard, "Patterns of Knowledge in API Reference Documentation," IEEE Transactions on Software Engineering, vol. 39, no. 9, pp. 1264-1282, Sept 2013. doi: 10.1109/TSE.2013.12. [Online]. Available: http://dx.doi.org/10.1109/ TSE.2013.12

[11] L. Shi, H. Zhong, T. Xie, and M. Li, "An Empirical Study on Evolution of API Documentation," in Proceedings of the 14th International Conference on Fundamental Approaches to Software Engineering: Part of the Joint European Conferences on Theory and Practice of Software, ser. FASE'11/ETAPS'11. Berlin, Heidelberg: Springer-Verlag, 2011. doi: 10.1007/978-3-642-19811-3_29. ISBN 9783-642-19810-6 pp. 416-431. [Online]. Available: http://dx.doi.org/10. 1007/978-3-642-19811-3_29 